\title{
2 Evolution der medizinischen Fortbildung durch Blended Learning und Mobile Learning
}

Jörg Ansorg

\section{Status der medizinischen Weiter- und Fortbildung in Deutschland}

Beide Phasen des postgraduierten kontinuierlichen Lernens in der Medizin sind durch Verordnungen der Bundesärztekammer reguliert (BÄK 2015a; BÄK 2013a). Während die Weiterbildung im ärztlichen Bereich die Phase zur Erlangung von Facharztqualifikationen beschreibt, ist die medizinische Fortbildung durch lebenslanges Lernen und kontinuierliche Aktualisierung von Wissen und Fertigkeiten geprägt.

Klassische Fortbildungsformate für Ärzte sind neben dem Studium von Fachliteratur und Fachzeitschriften der Besuch von Kongressen, Seminaren und Workshops. In den letzten beiden Jahrzehnten haben sich zusätzlich elektronische Fortbildungsformate entwickelt. Besonders beliebt sind dabei die CME-Beiträge in Fachzeitschriften, auch wenn es sich dabei in der Regel nicht um E-Learning, sondern um digitale Kopien von Printbeiträgen handelt.

Ein relativ neues Format stellen strukturierte curriculare Fortbildungen (BÄK 2015b) dar, die inhaltlich von der Bundesärztekammer in Kooperation mit Fachgremien definiert werden und mit einer besonderen Qualifikation abschließen. Das Curriculum gibt Inhalte, Lernziele und die aufzubringende Lernzeit exakt vor. Ziel dieser häufig interdisziplinären Fortbildungsangebote ist die Entwicklung spezifischer Fachkompetenzen. 


\section{Beispiele für strukturierte curriculare Fortbildungen}

- Krankenhaushygiene (200 Stunden)

- Hygienebeauftragter Arzt (40 Stunden)

- medizinische Begutachtung (64 Stunden)

- osteopathische Verfahren (160 Stunden)

- Ernährungsmedizin (100 Stunden)

Für viele strukturierte curriculare Fortbildungen sind kombinierte Lernangebote aus Präsenz- und E-Learning-Phasen (sogenanntes Blended Learning) möglich. Der maximal gestattete E-Learning-Anteil schwankt je nach Curriculum zwischen 25-50\%. Die Bundesärztekammer hat außerdem Qualitätskriterien für E-Learning-Fortbildungen aufgestellt (BÄK 2013b) und vergibt bei Einhaltung dieser Kriterien das Qualitätssiegel E-Learning sowie zusätzliche Fortbildungspunkte.

\section{Individualisierte Fortbildung durch Blended Learning}

Medizinische Fortbildungsangebote in Form von Blended-Learning zeichnen sich durch eine abgestimmte Kombination aus E-Learning und Präsenzphasen aus.

Der größte Vorteil dieser Lernmethode ist die Reduktion der Präsenzzeit. Mit Blended Learning wird außerdem das Kursniveau durch Harmonisierung des Vorwissens aller Teilnehmer angehoben. In der Präsenzphase bleibt dann mehr Zeit für Diskussionen und praktische Übungen sowie die aktive Einbindung der Kursteilnehmer.

\section{Vorteile von Blended Learning in der medizinischen Fortbildung}

- Reduktion der Präsenzzeit

- Effizienzmaximierung der Präsenzphase

- Individualisierung der Lerntempos in der E-Learning-Phase

- Steigerung von Motivation und Lernerfolg

Bei strukturierten curricularen Fortbildungsmaßnahmen erscheint die Entwicklung von Blended-Learning-Angeboten aufgrund des erheblichen Zeitaufwandes besonders lohnend.

Wesentlich für die Akzeptanz und den Erfolg neuer Lernmethoden ist der unmittelbar spürbare Nutzen für den Anwender sowie ein konsistentes didaktisches Konzept. Hierbei geht es nicht nur um den unmittelbaren Kosten- und Zeitvorteil einer reduzierten Präsenzphase, sondern auch um die Möglichkeit, das Lerntempo selbst zu bestimmen, Inhalte zu rekapitulieren und nach Abschluss des Kurses im Berufsalltag weiterhin auf die elektronischen Lerninhalte zuzugreifen.

Im Unterschied zu Lehrbüchern oder deren digitalen Kopien schaffen adaptive ELearning-Systeme in Kombination mit einer interaktiven Präsenzphase eine hochgradig individualisierte Lernsituation, die in der Erwachsenenbildung entscheidend für Lernerfolg und nachhaltige Kompetenzentwicklung ist. En passant motiviert ein 
derart individualisiertes Fortbildungspaket die Teilnehmer, am Ball zu bleiben, den Kurs engagiert durchzuarbeiten, mit eigenen Fragen und Beiträgen zu bereichern und sich in der Präsenzphase aktiv einzubringen.

\title{
Praxisbeispiel - Blended-Learning-Fortbildung von Hygienebeauftragten Ärzten (HBA) und Pflegekräften (HBP)
}

Der Bedarf an Hygienebeauftragten im ärztlichen und pflegerischen Dienst hat durch gesetzliche Vorgaben in den vergangenen Jahren erheblich zugenommen. Das aktualisierte Infektionsschutzgesetz (IfSG 2011: http://www.gesetze-im-internet.de/ifsg/ index.html) sowie die Länderhygieneverordnungen fordern Hygienebeauftragte Ärzte in nahezu jeder Krankenhausabteilung sowie in allen Praxen, die operative Eingriffe durchführen. In vielen Bundesländern besteht außerdem die Pflicht zum Vorhalten von Hygienebeauftragten Pflegekräften sowie Hygienebeauftragten Medizinischem Fachpersonal (MFA).

Der im ambulanten Sektor sprunghaft gestiegene Schulungsbedarf lässt sich seit 2014 durch die klassischen Fortbildungsangebote nicht mehr decken und ist aufgrund des erheblichen Zeitaufwandes (1 Woche Abwesenheit) für viele niedergelassene Ärzte nicht zumutbar.

Der Berufsverband der Deutschen Chirurgen (BDC e.V.) entwickelte deshalb auf Grundlage seiner langjährigen E-Learning-Erfahrung 2014 ein erstes Blended-Learning-Angebot zur curricularen Fortbildung zum Hygienebeauftragten Arzt (Kramer al. 2015; Kramer et al. 2016). Dieses wurde eng mit der Bundesärztekammer und einzelnen Landesärztekammern abgestimmt und der maximale E-Learning-Anteil im Curriculum auf $50 \%$ angehoben.

\author{
Blended-Learning-Fortbildung zum Hygienebeauftragten Arzt (HBA) \\ - klassisches E-Learning (20 Stunden) \\ - Abschlusskolloquium über 2 Tage inkl. Abschlusstest (20 Stunden) \\ - Aktualisierungen und Schulungsmaterialien für Nachgeordnete
}

Seit Ende 2014 ist dieses Blended-Learning-Angebot des BDC auf dem Markt und wurde kontinuierlich evaluiert. Hierbei wurde neben dem Standardfragebogen für ärztliche Fortbildung ein gemeinsam mit der Ärztekammer Westfalen-Lippe und der Bundesärztekammer entwickelter Test eingesetzt, um Nutzerverhalten und Verbesserungspotenzial detailliert zu erfassen.

Das Fortbildungsangebot wurde von Beginn an intensiv nachgefragt und wird seit Anfang 2015 auch an andere Facharztverbände lizensiert. Heute wird es von nahezu allen fachärztlichen Berufsverbänden als Alternative zur 40-stündigen Präsenzfortbildung empfohlen und angeboten. 


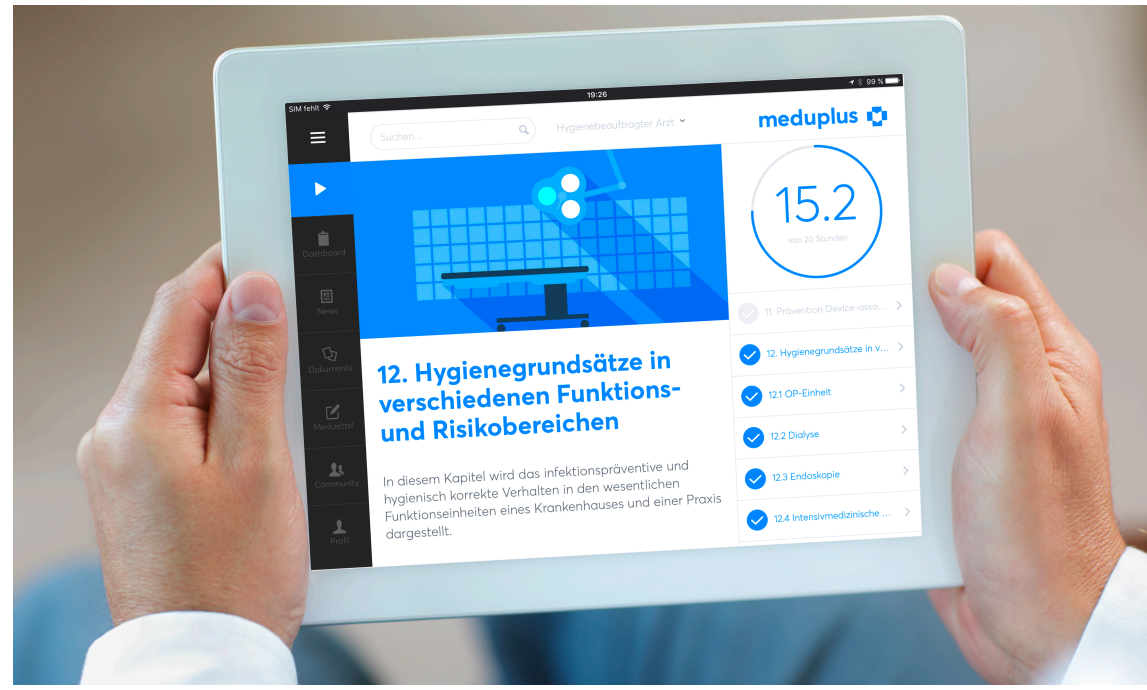

Abb. 1 Hygienegrundsätze in verschiedenen Funktions- und Risikobereichen, Meduplus ${ }^{\circledR}$

\section{Evolution der medizinischen Fortbildung mit der Meduplus Smart Learning ${ }^{\circledR}$ Methode}

Trotz positiver Resonanz und hoher Nachfrage zeigte die Evaluation des E-LearningTeils deutliches Verbesserungspotenzial auf. Von vielen Teilnehmern wurde unter anderem die mäßige Strukturierung und Nutzerfreundlichkeit des klassischen ELearning-Ansatzes kritisiert. Relevante Inhalte konnten nur schlecht von Zusatzinformationen und vertiefenden Referenzen differenziert werden, die Wissensfragen am Ende jedes Moduls boten zu wenig Interaktion und Erfolgserlebnisse.

Die große Teilnehmerzahl ( $\mathrm{n}=778$ ) und die für Ärzte typische Altersverteilung (Durchschnittsalter 55 Jahre) erlaubte bislang unbekannte Einblicke in die Erwartungen und Wünsche von erfahrenen Fachärzten an moderne elektronische Lernformate. Bei ca. der Hälfte der Teilnehmer identifizierten wir Verbesserungspotenzial und entschlossen uns bereits Mitte 2015, den E-Learning-Kurs komplett neu aufzusetzen.

In einem Joint-Venture unter der Marke Meduplus ${ }^{\circledR}$ wurde die Entwicklung eines völlig neuen E-Learning-Konzepts für die medizinische Fortbildung mit neuem didaktischen Ansatz sowie einer an die Zielgruppe adaptierten Nutzerführung vorangetrieben und von einem Team aus medizinischen Experten gemeinsam mit Didaktikern, Grafikern, Userinterface- und Web-Designern umgesetzt (s. Abb. 1).

\section{Meduplus Smart Learning ${ }^{\circledR}$ zum Hygienebeauftragten Arzt (HBA)}

- Wechsel zwischen Wissensvermittlung und -Assessment

- Individualisierung durch ein adaptives Lernsystem mit

- Belohnung von Vorwissen und Fokus auf Wissenslücken

- Learning Community zum Austausch der kontinuierlichen Verbesserung

- Reduktion der Präsenzphase von 5 auf 2 Tage bei gesteigerter Lerneffizienz 


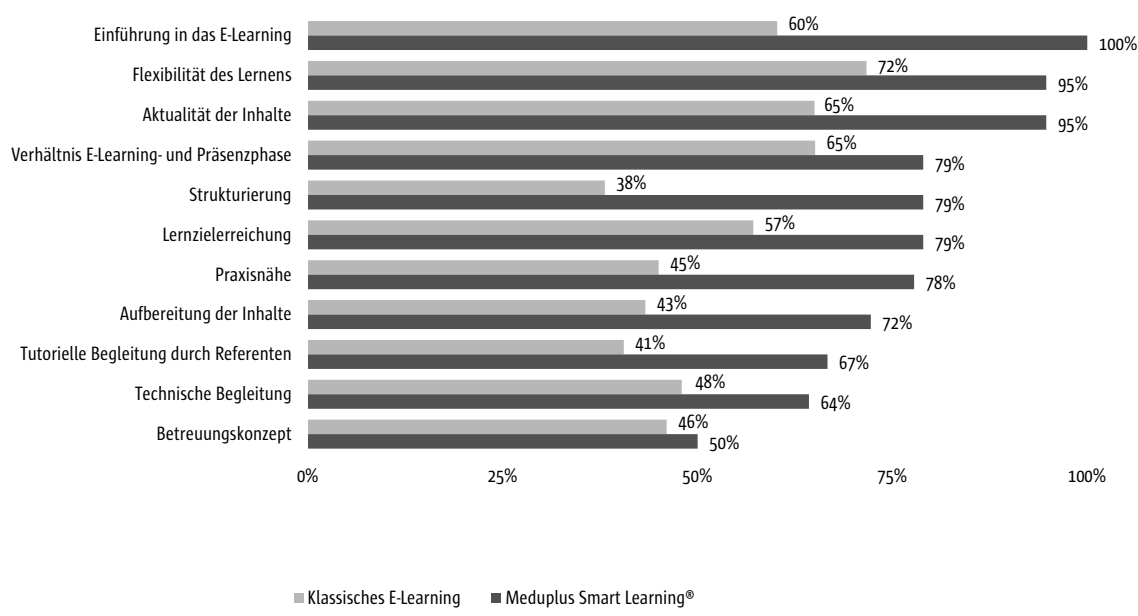

Abb. 2 Evaluation der E-Learning-Module mit "gut" und "sehr gut"

Mit Meduplus Smart Learning ${ }^{\circledR}$ gelingt es erstmals in der medizinischen Fortbildung, Vorwissen zu belohnen und auf das Schließen individueller Wissenslücken zu fokussieren. Damit vermittelt das System Freude am Lernen und dem eigenen Lernfortschritt. Die Mitgliedschaft in einer exklusiven Learning Community schafft Verbundenheit und ermöglicht es jedem Teilnehmer, am Wissen anderer Nutzer zu partizipieren oder das Experten- und Tutorenteam zu kontaktieren.

Das neue E-Learning-System wurde im 3. Quartal 2016 eingeführt und sofort vom Markt angenommen. Die Resonanz war sehr positiv und wurde nach denselben Maßstäben wie das vorangegangene System evaluiert. Die Ergebnisse zeigten eine erhebliche Verbesserung in allen Erhebungskriterien (s. Abb. 2). Die Nutzerzufriedenheit insgesamt konnte von $48 \%$ auf $78 \%$ gesteigert werden.

Technisch wurde das Gesamtsystem für die Nutzung mit mobilen Endgeräten (Smartphones und Tablets) optimiert. Dies gestattet nicht nur das bequeme Lernen an jedem Ort sondern qualifiziert das Lernpaket als interaktives und mobiles Referenzwerk für den Klinik- und Praxisalltag.

\section{Fazit}

Blended Learning hat das Potenzial, die medizinische Fortbildung in Zeiten dünner Personaldecken und Zeitbudgets zu revolutionieren. Werden Didaktik und Design neu gedacht und mit einem adaptiven Lernsystem kombiniert, wird ärztliche Fortbildung auf eine neue Stufe gehoben. Bei diesem Ansatz steht der nachhaltige Kompetenzerwerb bei gleichzeitig reduziertem Ressourcenverbrauch im Mittelpunkt und schafft Mehrwert für alle Beteiligten, nicht zuletzt für Krankenhausträger und Patienten.

\section{Literatur}

Bundesärztekammer (BÄK) (2013a) (Muster-)Fortbildungsordnung der Bundesärztekammer: http://www. bundesaerztekammer.de/aerzte/aus-weiter-fortbildung/fortbildung/ (abgerufen am 28.07.2017) 
Bundesärztekammer (BÄK) (2013b) Qualitätskriterien elearning der Bundesärztekammer: http://www.bundesaerztekammer.de/fileadmin/user_upload/downloads/KritELearningVg.pdf (abgerufen am 28.07.2017)

Bundesärztekammer (BÄK) (2015a) (Muster-)Weiterbildungsordnung und (Muster-)Richtlinien der Bundesärztekammer: http://www.bundesaerztekammer.de/aerzte/aus-weiter-fortbildung/weiterbildung/musterweiterbildungsordnung/ (abgerufen am 28.07.2017)

Bundesärztekammer (BÄK) (2015b) Strukturierte curriculare ärztliche Fortbildungen der Bundesärztekammer: http://www.bundesaerztekammer.de/aerzte/aus-weiter-fortbildung/fortbildung/strukturierte-curriculare-fortbildung/ (abgerufen am 28.07.2017)

Kramer A, Bartels C, Ansorg I, Großkopf V, Schanz M (2015) Hygienefachpersonal in der ambulanten und stationären Versorgung - ein bundesweiter Überblick am Beispiel des Hygienebeauftragten Arztes (HBA). Rechtsdepesche für das Gesundheitswesen, Jul/Aug 2015, 158-163

Kramer A, Bartels C, Hoffmann R, Ansorg I (2016) Verantwortung hygienebeauftragter Ärzte für die Prävention nosokomialer Infektionen. Trauma und Berufskrankheit 17, 3/2016, Springer Verlag Heidelberg 